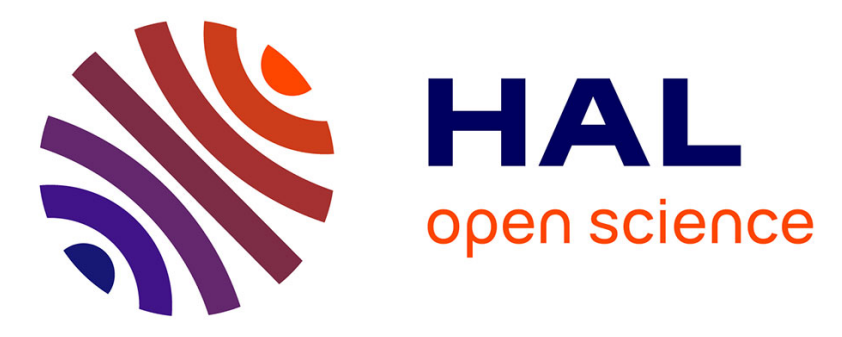

\title{
A generalized white-patch model for fast color cast detection in natural images
}

Jose Luis Lisani, Ana Belen Petro, Edoardo Provenzi, Catalina Sbert

\section{To cite this version:}

Jose Luis Lisani, Ana Belen Petro, Edoardo Provenzi, Catalina Sbert. A generalized white-patch model for fast color cast detection in natural images. IS\&T International Symposium on Electronic Imaging - Retinex at 50: Image Processing, Feb 2016, San Francisco, CA, United States. hal-01386173

\section{HAL Id: hal-01386173 https://hal.science/hal-01386173}

Submitted on 23 Oct 2016

HAL is a multi-disciplinary open access archive for the deposit and dissemination of scientific research documents, whether they are published or not. The documents may come from teaching and research institutions in France or abroad, or from public or private research centers.
L'archive ouverte pluridisciplinaire HAL, est destinée au dépôt et à la diffusion de documents scientifiques de niveau recherche, publiés ou non, émanant des établissements d'enseignement et de recherche français ou étrangers, des laboratoires publics ou privés. 


\title{
A generalized white-patch model for fast color cast detection in natural images
}

\author{
Jose Luis Lisani, University of Balearic Islands, Palma de Mallorca, Spain \\ Ana Belén Petro, University of Balearic Islands, Palma de Mallorca, Spain \\ Edoardo Provenzi, Laboratoire MAP5 (UMR CNRS 8145), Université Paris Descartes, Sorbonne Paris Cité, France \\ Catalina Sbert, University of Balearic Islands, Palma de Mallorca, Spain
}

\begin{abstract}
We present a generalized white-patch technique able to rapidly detect color cast of natural images. Instead of relying on the chromatic information of a single perfectly reflective patch in the image, as pure white-patch models do, we consider a connected region of pixels that will serve as white reference for the method. The pixels belonging to the white reference region must comply with three properties: 1) they do not have to be completely saturated; 2) they must belong to the p\% of pixels with brightest intensity in the whole image (where $p$ is a parameter of the model); 3) the area of the connected region formed by these pixels must overcome a threshold of significance A (a second parameter). Color cast is detected if the average intensity in the three separated chromatic channels $R G B$ is distant enough from a neutral grey level, where the distance is measured through an angular metric.
\end{abstract}

\section{Introduction}

Color cast is generated by the presence of a non neutral illuminant in a visual scene [2]. The effect of a color cast is the appearance of a colored layer superimposed to the entire image.

This layer can be a pleasant effect induced by purpose by a photographer, but it may also be an unwanted problem for many applications.

To quote just an example, the tracking of an object in large environments (e.g. a stadium or a subway station) can fail dramatically due to the fact that the color cast can change significantly the chromatic information of the tracked object.

Humans, on the contrary, thanks to a property called color constancy, have the ability to perceive colors in a much more stable way than machines. In fact, a human being embedded in a visual scene and adapted to its illuminant conditions is able to automatically discount a large part of the color cast.

It is thus important to provide digital devices with an automatic color cast detection and removal mechanism, this is still an open issue in image processing and computer vision. In the survey [4], the aim of computational color constancy is defined as: 'The goal [...] is to correct the target images (under different colored light sources), so that they appear identical to the canonical image (under a white light source)'.

The main reason underlying the difficulty of this task is that, without having prior information on the content of a photographed scene, it has been proved to be extremely difficult to understand if an image is affected by a color cast or if it is characterized by a dominant color.
A clarifying example is provided by close ups: if we take the close up of, say, the yellow petals of a flower under the daylight, then (a correctly exposed) digital picture will not be affected by color cast and it would be typically characterized by large red and green values and much smaller blue values in every pixel of the image. This is the same qualitative scenario that one would have with a picture affected by a yellow color cast, as can be seen in Figure 1.
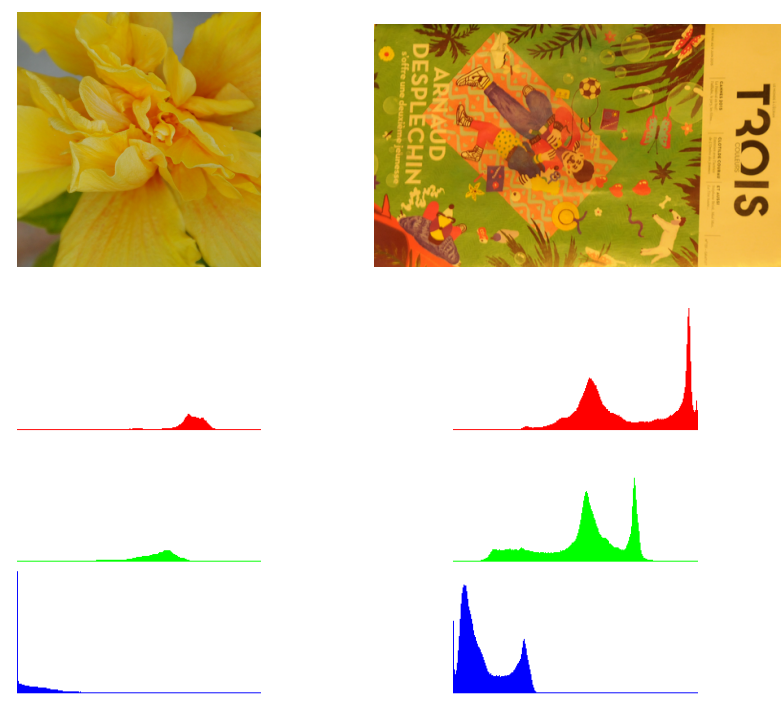

Figure 1. Left: Image without color cast and its RGB histograms. Right: Image affected by color cast and its RGB histograms. In both cases, for two different reasons, the $R$ and $G$ values dominate the $B$ values.

Following the definition in [4], the method that we propose here falls in the category of Low-Level Statistics-Based Methods.

The aim of this work is to provide a reliable and fast method to detect images with color cast without any prior information on the image content, in the same spirit as [3], even if the techniques used here are different from those used in that paper.

Given a dataset of digital pictures, we want to be able to point out all the picture affected by color cast. A challenge is given by the previously discussed ambiguity between images taken under non-neutral illuminants and pictures characterized by large areas with a homogeneous dominant color. We aim at minimizing the misinterpretations due to this ambiguity, underlying until what extent this is possible. 


\section{The proposed algorithm}

We propose the following algorithm to assess the presence/absence of color cast in a digital image. In what follows, we assume that the $R G B$ values of the image are in the discrete range $\{0, \ldots, 255\}$.

1. Detect saturated pixels, defined as pixels whose $R, G$ or $B$ values are above an upper threshold $T$. These saturated pixels are discarded in the following computations.

2. Detect "bright" pixels, defined as the $p \%$ of pixels with highest intensity values. Here the intensity value of a pixel is defined as the arithmetic average of $R, G$ and $B$.

3. Find connected regions of bright pixels. The goal is to find a relatively large group of pixels that will be considered as the "reference white" of the image, therefore, all the connected regions with less than $A$ pixels are discarded.

4. Compute the average $R, G$ and $B$ values of the pixels belonging to the remaining regions after step 3 , if any.

5. If no color cast is present in the image, this average $R G B$ vector should be aligned with the "ideal white" vector of the $R G B$ color cube, that is, the achromatic axis, i.e. the diagonal of the cube connecting $(0,0,0)$ to $(255,255,255)$. The angular difference ${ }^{1}$ between these two vectors gives a measure of the departure from neutral illumination in the input image. A threshold $\alpha$ on this angle determines the absence or presence of color cast.

\section{Experimental Results}

The algorithm proposed in the previous section assumes that there exists a threshold $\alpha$ for the angle between the estimated illuminant of a scene and the achromatic axis, that permits to discern whether the scene is affected by color cast or not.

In order to test this hypothesis and to give an initial estimate of this threshold we have built a small database, given by 90 natural pictures taken under daylight and unknown artificial illuminants, to reproduce the most common situation that a photographer may experience. The camera used to build the dataset is a reflex Nikon D300 and the pictures were taken in RAW format, converted to jpeg with the choice of minimum amount of compression.

In Figure 2 and Figure 3 we show two excerpts of our database with and without color cast, along with the corresponding connected white reference regions and the angle of deviation from the achromatic axis.

We found that with the following choice of parameters, $T=$ $250, p=1, A=20, \alpha=10^{\circ}$, the algorithm was able to point out all images in our dataset affected by color cast, with no exception.

However, we got also a few false positives, as with the close up of the flower with yellow petals shown in Figure 1-left. In this case we got an angle of $15^{\circ}$, slightly above the threshold. As with any method relying on fixed thresholds there is always the possibility of misclassifications, specially in the case of images with dominant colors. In these cases we believe that contrast plays a fundamental role: even for human beings it is impossible to decide if a perfectly uniform surface is affected by color cast or it has a dominant color without a priori information about the surface.

\footnotetext{
${ }^{1}$ The use of an angular distance is quite common in computational color constancy, see e.g. [4] and [5].
}

However, as soon as contrast information increases, our color constancy abilities come into play and we are able to strongly reduce the effects of the possible presence of a color cast. The question of how small this contrast must be is clearly an important one from both a theoretical and a computational point of view. This shall be the subject of further research and it is beyond the scopes of the present paper.

In order to exhaustively test our algorithm we used the 11346 images in Ciurea's dataset [1], for which the true value of the illuminant is available (it is computed by taking as reference the colors in a gray ball present in all the images). For 10732 of these images our method was able to correctly estimate the presence or absence of a color cast. For the remaining part of the database, around the $6 \%$ of the whole set, the bright pixels distribution in the images were under the threshold of significance $A$ to allow the formation of meaningful connected regions of pixels considered as a reference for white.

The database contains images with different levels of color cast: outdoor scenes without color cast, outdoor scenes with color cast due to sunset light, indoor scenes with weak cast and indoor scenes with strong cast caused by artificial illumination. Figure 4 shows examples of these images. Below each image the angles between the true illuminant and estimated illuminants with the achromatic axis are displayed. The values are similar in all cases. Moreover, as expected, higher values of the angle correspond to images with stronger color cast.

The similarity between the estimated illuminant and the true value provided by the database was tested by computing the angular differences between both angles. The results are displayed in Table 1 and Figure 5 and they show that for $60 \%$ of the images the difference between the estimated value and the actual one is small (less than $4.75^{\circ}$ ), and only for $10 \%$ of the images the difference
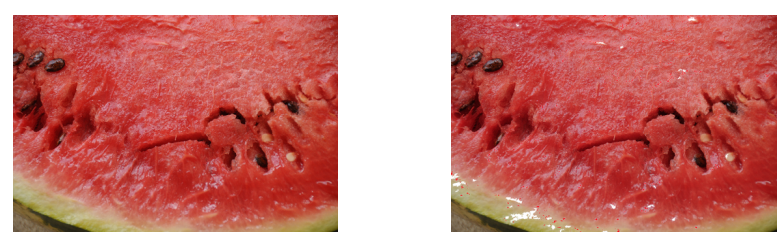

Figure 2. Left: original "watermelon" image taken under daylight. Right: connected regions of white reference. In spite of the fact that the image is characterized by a dominant color, there is enough contrast to let our algorithm detect white reference regions with a deviation angle with respect to the achromatic axis of $7.45^{\circ}$, below the threshold $\alpha=10^{\circ}$. Thus, the image is correctly characterized as not being affected by color cast.
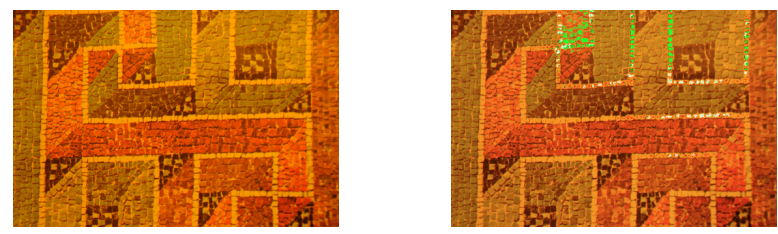

Figure 3. Left: original "Ravenna mosaic" image taken under unknown yellow illuminant. Right: connected regions of white reference. The angular deviation from the achromatic axis is $37.21^{\circ}$, much above the threshold $\alpha=$ $10^{\circ}$. Thus, the image is correctly identified as being affected by color cast. 


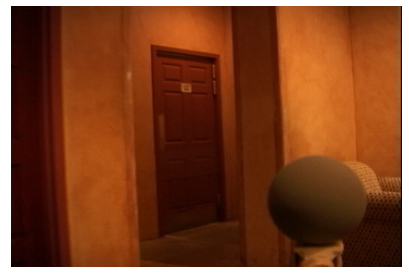

$25.5,28.75$

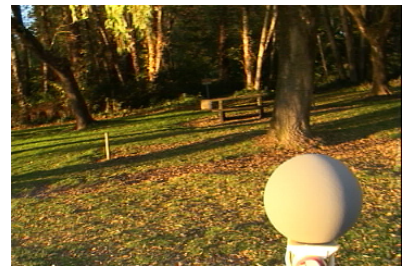

$11.48,10.82$

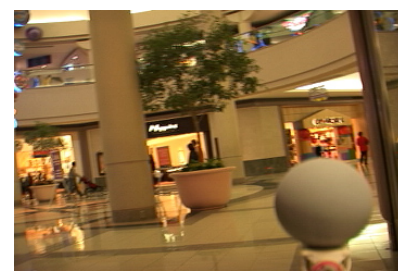

$12.09,10.50$

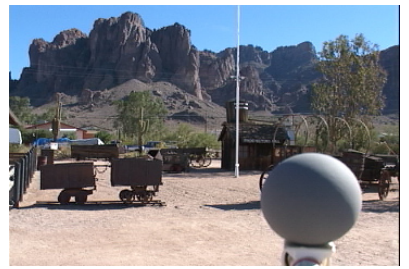

$1.50,0.87$
Figure 4. Examples of indoor images (top row) with strong (left) and weak (right) color cast. In the latter case both natural and artificial illumination are present in the scene. Bottom row, outdoor images with (left) and without color cast (right). In this case the color cast is due to the sunset light. The number below each image are, respectively, the angle between the given illuminant and the achromatic axis and the angle between the estimated illuminant and the achromatic axis. The values are similar in all cases. Moreover, we observe that higher values of the angle correspond to images with stronger color cast.

is above $11.04^{\circ}$.

Table 1: Deciles of the distribution of angular differences shown in Figure 5. These values correspond to 10732 out of the 11346 images in the dataset. For the rest of images our algorithm was unable to find a large enough patch to estimate the illuminant.

$\begin{array}{cccccc}0 \% & 10 \% & 20 \% & 30 \% & 40 \% & 50 \% \\ 0.01 & 0.63 & 1.24 & 1.98 & 2.76 & 3.67 \\ & & & & & \\ 60 \% & 70 \% & 80 \% & 90 \% & 100 \% & \\ 4.75 & 6.04 & 7.90 & 11.04 & 29.26 & \end{array}$

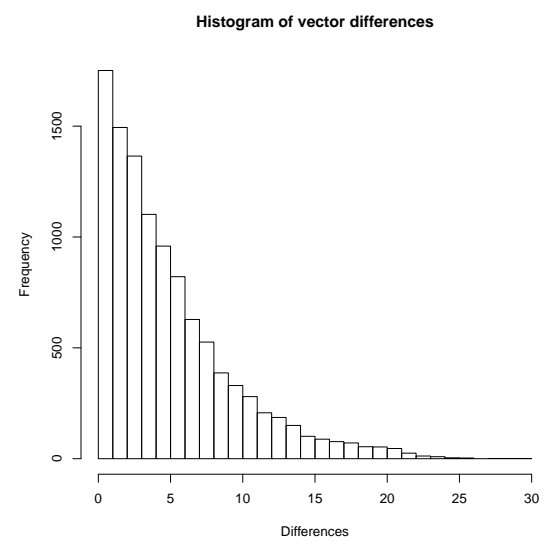

Figure 5. Histogram of angular differences between estimated illuminant vectors and actual values in Ciurea's Dataset [1].
Even in the cases where the difference between both vectors was large. it is not clear that our method really provides a wrong result. The image in Figure 6-left clearly exhibits a color cast, even if the illuminant in the dataset differs only $4.74^{\circ}$ from the achromatic axis. Our method correctly detects the cast, since the estimated angle $\left(12.73^{\circ}\right)$ is above the threshold $10^{\circ}$. On the other hand, for the image in Figure 6-middle, the illuminant in the database shows a high deviation from the achromatic axis $\left(20.85^{\circ}\right)$, while our method gives $5.60^{\circ}$. The reason is there are indeed two illuminants in the scene, a first illuminant near the camera (where the reference gray ball is placed) which exhibit a color cast, and a neutral illuminant (natural light) on the left side of the picture, which is the one detected by our method. Finally, for the image in Figure 6-right, the illuminant in the database also shows a high deviation from the achromatic axis $\left(15.96^{\circ}\right)$ but clearly the image has not color cast. For this image our method gives $0.57^{\circ}$, which is more coherent in this case.

In general, we observed that for most of the images with color cast the angle is above $10^{\circ}$ while below this threshold most of the images are free of color cast. Some exceptions to this rule can be observed in Figure 7 and shall be the subject of further research. The result in Figure 7-left may be due to the presence in the scene of both natural and artificial illumination.
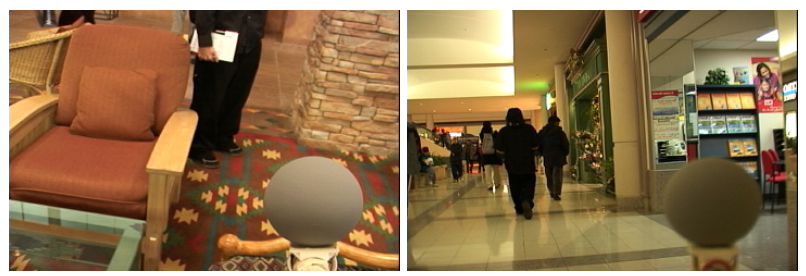

Figure 7. Two examples that do not agree with the rule that classifies images as without color cast when the angle of the estimated illuminant is below $10^{\circ}$. The values of the estimated angles are, respectively, $7.38^{\circ}$ and $5.59^{\circ}$.

\section{References}

[1] F. Ciurea And B. Funt, A large image database for color constancy research, in Imaging Science and Technology Eleventh Color Imaging Conference, 2003, pp. 160-164.

[2] M. EbNer, Color Constancy, The Wiley-IS\&T Series in Imaging Science and Technology, Wiley, 2007.

[3] F. Gasparini AND R. Schettini, Color balancing of digital photos using simple image statistics, Pattern Recognition, 37 (2004), pp. $1201-1217$.

[4] A. GijseniJ, T. Gevers, And J. van De Weijer, Computational color constancy: Survey and experiments, Image Processing, IEEE Transactions on, 20 (2011), pp. 2475-2489.

[5] J. Vazquez-Corral, C. A. Parraga, M. Vanrell, and R. BALDRICH, Color constancy algorithms: Psychophysical evaluation on a new dataset, Journal of Imaging Science and Technology, 53 (2009).

\section{Author Biography}

Jose-Luis Lisani received the PhD degree in Computer Science and Applied Mathematics by the Universities of Illes Balears (Spain) and ParisDauphine (France) in 2001. He is currently Assistant Professor at the University of Illes Balears (Spain). His research interests include the 

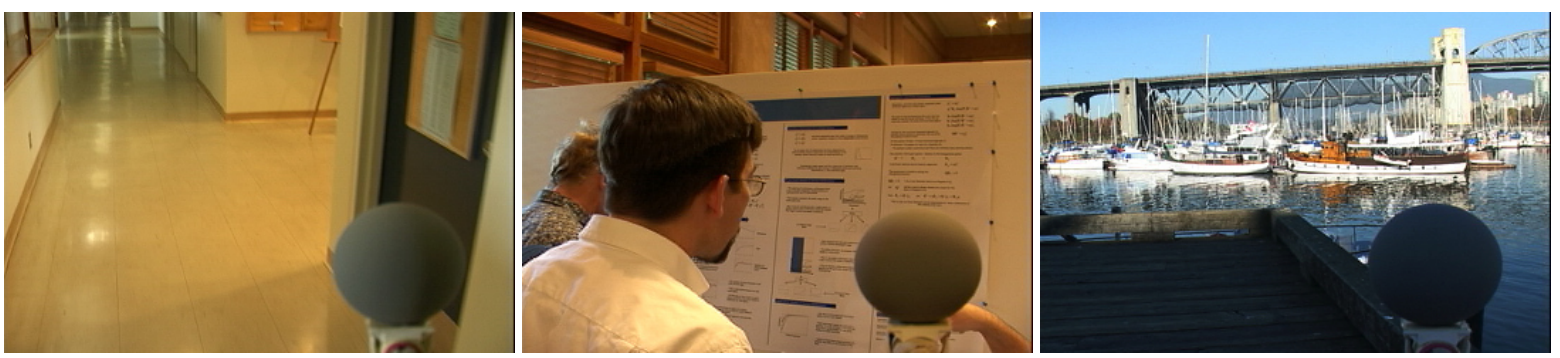

Figure 6. Three examples were the estimated illuminant differs considerably from the "true" value furnished by the Ciurea's Dataset [1]. See text for comments.

analysis and processing of color images and video sequences. He has coauthored the book 'A Theory of shape identification' (Springer LNM, 2008).

Ana Belen Petro was born in Mallorca (Spain) in 1977. She defended her PhD thesis in color image analysis in 2006 at the University of Balearic Islands (Spain). She is currently an Assistant Professor at the University of Balearic Islands (Spain) and she collaborates with the CMLA laboratory at ENS Cachan (France). Her research interest includes color image processing and its mathematical analysis.

Edoardo Provenzi received the Master degree in Theoretical Physics from the University of Milan in 2000 and the PhD in Mathematics and Application from the University of Genoa in 2004. He is an Associate Professor at the Universite Paris Descartes, France in the laboratory of mathematics applied to image processing. His main field of research is color image processing, with particular interest in variational techniques and perceptual properties of human vision.

Catalina Sbert received the Master degree in Mathematics from the University Autonoma de Barcelona in 1987 and the PhD degree on Computer Science from the University of Illes Balears (Spain) in 1995. She is currently an Assistant Professor at the University of Illes Balears (Spain). Her research interest includes image processing, computer vision, and the applications of variational techniques and partial differential equations to both previous fields. 